\title{
ACRL members running for ALA Council in the spring 2020 election
}

The following ACRL members are either nominated or petition candidates for ALA councilor. ACRL members are encouraged to vote for these candidates to increase ACRL's voice in ALA affairs.

Tina Baich, Associate Dean for Collections, IUPUI University Library, Indianapolis

Regina M. Beard, Business Librarian, Florida Gulf Coast University, Fort Myers

Emily Bergman, Campus Librarian, Chicago School of Professional Psychology, Los Angeles

Latrice Booker, Dean of the Library, Indiana University Northwest, Gary

Matthew Ciszek, Library Director, Penn State Behrend, Erie

John DeSantis, Cataloging and Metadata Services Librarian, Dartmouth College, Hanover, New Hampshire

Yvonne Dooley, Business Librarian, University of North Texas, Denton

Cynthia Ellison Dottin, Adjunct Lecturer, FIU Honors College, Florida International University, Miami

Vicky Hart, Director of Library Services, Northeast Lakeview College, San Antonio, Texas

Peter Hepburn, Head Librarian, College of the Canyons, Santa Clarita, California

Shanna Hollich, Collections Management Librarian, Wilson College, Chambersburg, Pennsylvania

Del R. Hornbuckle, Dean of Library Services, California State University-Fresno

Grace Jackson-Brown, Research and Instruction Librarian, Missouri State University, Duane G. Meyer Library, Springfield

Robin Kear, Liaison Librarian, University of Pittsburgh, Pennsylvania

Oscar R. Lanza-Galindo, Library and Learning Commons Director, Bristol Community College, Fall River, Massachusetts

Binh P. Le, Librarian, The Pennsylvania State University, Abington, Pennsylvania
Rodney Lippard, Director, Gregg-Graniteville Library and the Center for Student Achievement, University of South Carolina-Aiken

Lesley Looper, Team Lead, Shelf Preparation Section, Resource Description Department, Duke University Libraries, Durham, North Carolina

Steve Matthews, Library Director Emeritus, Currier Library, Foxcroft School, Middleburg, Virginia

Joe Mocnik, Dean of Libraries, North Dakota State University, Fargo

Alanna Aiko Moore, Librarian for Sociology, Ethnic Studies, Gender Studies, University of California-San Diego, La Jolla

Leslie L. Morgan, Africana Studies Education Librarian, University of Notre Dame, Indiana

Jennifer Pate, Scholarly Communications and Instructional Services Librarian, University of North Alabama, Florence

Raymond Pun, Instruction/Research Librarian, Alder Graduate School of Education, Redwood City, California

Edward L. Sanchez, Head, Library Information Technology, Marquette University, Milwaukee, Wisconsin

John Sandstrom, Acquisitions Librarian, New Mexico State University, Las Cruces, New Mexico

Paul A. Sharpe, Dean of Libraries, University of Texas Rio Grande Valley, Edinburg-BrownsvilleHarlingen

Jim Teliha, Dean of the Library Learning Commons, Utica College, New York

Scott Walter, University Librarian and University Copyright Officer, Illinois Wesleyan University, Bloomington

Brad Warren, Associate Dean of Library Services, University of Cincinnati, Ohio

Angela A. Williams, MLK Librarian, Syracuse University, New York

Harriet Wintermute, Head, Metadata Services, Iowa State University Library, Ames 